\title{
Formation of Thin Current Sheets in the Solar Atmosphere
}

\author{
C. Z. Cheng and G. S. Choe ${ }^{1}$
}

Princeton Plasma Physics Laboratory, Princeton University, Princeton, NJ 08543, USA

\begin{abstract}
In a two-dimensional quadrupolar magnetic field geometry, it is demonstrated that various magnetohydrostatic equilibrium field configurations containing current sheets can be formed either by changes in thermal properties or by footpoint displacement. The shape of current sheets depends on the mass distribution in flux tubes. If the mass per flux tube is maximum at the center of the low-lying bipolar arcades, a current sheet is formed between these two arcades along the separatrix line as the plasma temperature (or the shearing footpoint displacement) is increased above a critical value. If the mass per flux tube is higher in the outer tubes of the bipolar arcades than in the inner ones, a current sheet with a sharp downward-pointed tip hanging at a distance above the bottom boundary is formed when the temperature is decreased below a critical value. Resistive magnetic reconnection in the latter type current sheet results in a magnetic island wrapped in dipped field lines, which is regarded as a plausible prominence field configuration.
\end{abstract}

\section{Introduction}

It is widely believed that magnetic reconnection processes are involved in solar eruptive phenomena, coronal heating and prominence formation. Due to a large magnetic Reynolds number $\left(\sim 10^{12-14}\right)$ in the solar atmosphere, a very thin current sheet is considered as a necessary condition of magnetic reconnection. Except during dynamic phases related to instabilities or magnetic reconnection, the solar plasma is in a near-equilibrium state because the wave transit time in coronal magnetic structures is short compared to the time-scale of external disturbances. When a system parameter is changed continuously in space and in time, the corresponding equilibrium may not exist in the initially given field topology, but may be found in a different topology. Under the ideal MHD conditions, such a transition of topology is inhibited and thus some field lines collapse to form a current sheet (see, e.g., Parker 1994). In this paper, we have constructed a sequence of $2 \mathrm{D}$ magnetohydrostatic (MHS) equilibria under the constraint of topology invariance and demonstrated the unavoidability of current sheet formation as system parameters, such as the plasma temperature and the footpoint displacement, are varied across critical values. In such current sheet configurations magnetic reconnection can be triggered by plasma dissipation to

\footnotetext{
${ }^{1}$ Presently at Tongmyong University of Information Technology, Pusan, Korea
} 
form either magnetic islands or an X-point magnetic field topology.

\section{Current Sheet Formation by Temperature Change}

In the solar corona, the pressure change in a flux tube is more likely to come from a temperature change than a change in the mass content because the photosphere is considered to be quite rigid. Thus, we take the invariance of the mass per flux tube as a constraint and choose the temperature as the control parameter. A magnetofrictional method (Chodura and Schlüter 1981, Choe and Lee 1996) is used to to find $2 \mathrm{D}$ equilibrium solutions for a given topology.

\subsection{Temperature Increase}

A quadrupolar potential field solution by Low (1992) is adopted as the initial configuration so that the density is a function of height only and the mass per flux is maximum at the center of the low-lying bipolar arcades. We obtained equilibrium solutions as the temperature is uniformly increased. Since the expansion of plasma is more effective in the outer flux tubes than in the inner ones, the resulting pressure profile at the base has a pressure maximum at the center of each bipolar regions. As the temperature is increased above a critical value, the plasma pressure gradient pushes out the field lines of each bipolar region so that a current sheet of a finite length is formed where the field lines from each bipolar region come into contact (see Figure 1). The lower tip of this current sheet touches the origin and the current sheet extends along the separatrix.
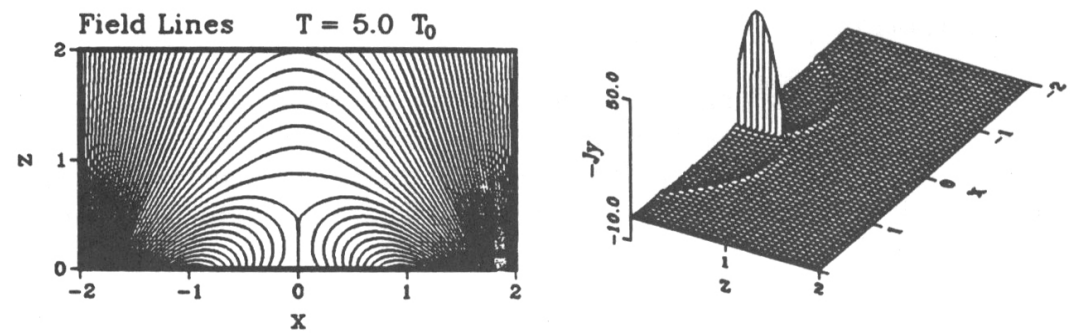

Figure 1. Formation of a current sheet by temperature increase. The field lines and the current density are shown.

\subsection{Temperature Decrease}

In this case, the pressure is higher in the outer tubes than in the inner ones in the initial equilibrium. While the potential field subject to the same boundary condition has an X-point, the initial equilibrium field has none because the higher mass in the outer flux tubes depresses the system (Low 1992). As the temperature becomes uniformly lower in the whole atmosphere, the pressure scale height decreases, and the magnetic field pressure dominates the plasma 
pressure in the upper part of the flux tubes. As the temperature is decreased below a critical value, the subsequent expansion of field lines creates a tangential discontinuity. The topology of the current sheet in this case is different from that in the former case in that a sharp-pointed tip of the current sheet hangs a distance above the bottom boundary (see Figure 2).
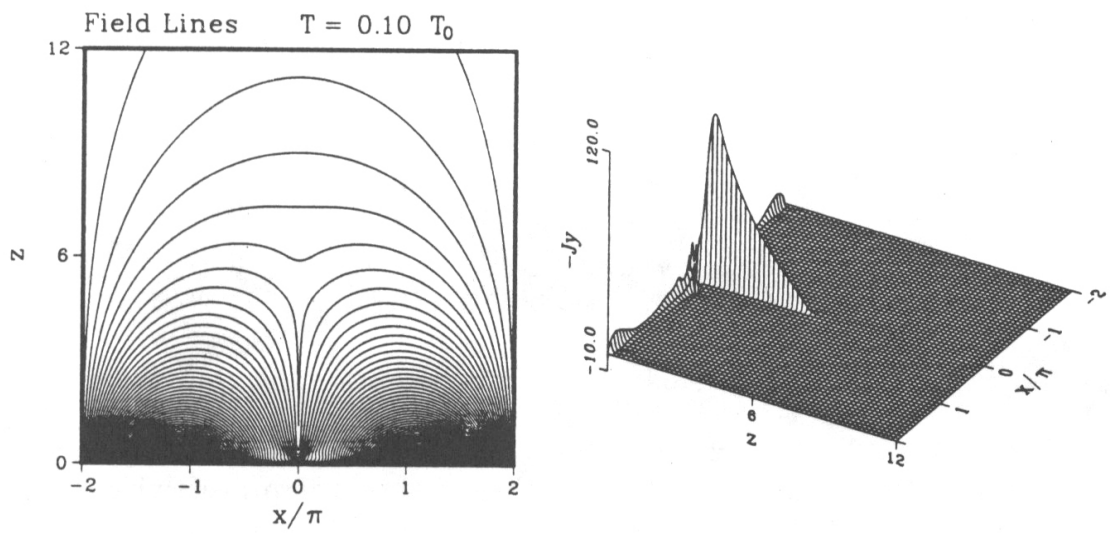

Figure 2. Formation of a current sheet by temperature decrease. The field lines and the current density are shown.

Starting with an equilibrium obtained with the temperature reduced to $1 / 4$ of the initial temperature, we performed resistive MHD simulations while lowering the temperature. Magnetic reconnection takes place in the current sheet and a vertically elongated magnetic island is formed in a thin current layer as shown in Figure 3. The density in the island is found higher than that in the surroundings. Although the study is based on a 2D isothermal model, it shows for the first time how a magnetic island can be formed between two bipolar regions. Such a field configuration was suggested by Malville (in a discussion reported by Anzer 1979) as a plausible model of prominence magnetic fields.

\section{Current Sheet Formation by Footpoint Displacement}

We have also studied the current sheet formation by a shearing footpoint motion. In a complex field topology, the footpoint displacement may not be continuous even though the shearing velocity profile is continuous. To avoid this trivial case, we make sure that the footpoint displacement is everywhere continuous. As the shear is increased in a low $\beta$ plasma, the two low-lying bipolar arcades expand outward and expel the magnetic flux belonging to the upper arcade. Above a critical amount of shear, a current sheet is formed between those two arcades and also along the separatrix field lines similar to that shown in Figure 1. 

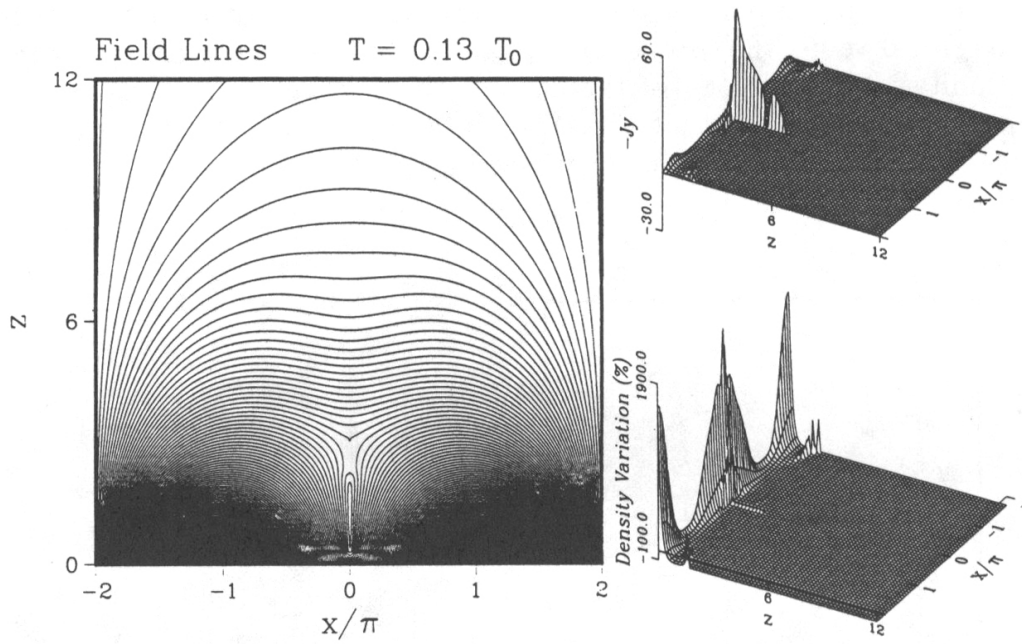

Figure 3. The field configuration, current density and density variation resulting from atmospheric cooling with nonzero resistivity. The magnetic island residing in a current layer should be noted.

\section{Conclusion}

In this paper, we have shown that formation of a current sheet can be quite common and ubiquitous in the solar atmosphere with a quadrupolar field geometry. Due to footpoint displacements and thermodynamic changes the system can evolve to form a current sheet. In addition to the possibility of a current sheet tied to the bottom boundary, a current sheet can also be suspended above the bottom boundary as long as a large enough mass exists around its tip. Magnetic reconnection in this current sheet can lead to a magnetic field configuration favorable for prominence formation.

Acknowledgments. This work is supported by NSF grant ATM-9696232 and DoE Contract No. DE-AC02-76-CHO3073.

\section{References}

Anzer, U. 1979, in Physics of Solar Prominences, E. Jensen, P. Maltby and F. Q. Orrall, Institute of Theoretical Astrophysics: Blindern-Oslo, p. 322

Chodura, R., and Schlüter, A. 1981, J. Comp. Phys., 41, 68

Choe, G. S., and Lee, L. C. 1996, ApJ, 472, 360

Low, B. C. 1992, A\&A, 253, 311

Parker, E. N. 1994, Spontaneous Current Sheets in Magnetic Fields, Oxford University Press: Oxford 